\title{
Reflectance confocal microscopy: new micromorphological insights into inflammatory skin diseases
}

In this issue of the BJD, Ardigo et al. and the International Confocal Working Group (ICWG) present an extensive and well-performed study on noninvasive imaging of superficial inflammatory skin diseases, focusing on defining diagnostic criteria for distinction between the three main groups: psoriasiform, spongiotic and interface dermatitis. ${ }^{1}$ Reflectance confocal microscopy (RCM) was used for obtaining highresolution in vivo images of superficial skin layers.

Over the last decade, RCM has become a well-established method for diagnosing melanocytic lesions. It can now distinguish between benign naevi and malignant melanomas with a high level of accuracy. ${ }^{2}$ Another field for RCM is epithelial skin cancer. $^{3,4}$ For both melanocytic tumours and nonmelanoma skin cancer, numerous studies have been published to define diagnostic criteria, pitfalls, sensitivity and specificity for RCM. These include a scoring system for melanomas, as well as diagnostic algorithms for the differentiation between benign naevi and melanomas.

In addition to utility in the diagnosis of skin tumours, RCM also offers insight into dynamic changes within the skin. For example, treatment of actinic keratoses can be monitored and controlled over time. ${ }^{5}$ Furthermore, blood flow and vessel morphology give additional information for diagnosing basal cell carcinomas. More recently, RCM has been studied as a diagnostic tool in onychomycosis, ${ }^{6}$ demodex $^{7}$ and scabies.

For inflammatory skin diseases with characteristic clinical features, diagnosis can be made with the naked eye. Nevertheless, in some cases, biopsies are necessary to confirm the diagnosis and to rule out possible similar-looking differential diagnoses. There are already case reports and studies published using RCM in inflammatory skin diseases ${ }^{8}$; however, systematic studies for determination of characteristic features have so far been lacking. This is starting to change: RCM findings in allergic contact dermatitis are different from those found in irritant dermatitis ${ }^{9}$; psoriasis characterized using RCM images features elongated papillae, fast blood flow and prominent thickening of the epidermis ${ }^{10}$; cutaneous lupus erythematosus and mycosis fungoides have also been evaluated by RCM.

Ardigo et al. present a large multicentre study of the International Confocal Working Group on defining diagnostic criteria for inflammatory diseases and developing a diagnostic algorithm. ${ }^{1}$ Twenty-one authors from 19 institutions in nine countries participated in this research. Only experienced RCM users were included, following a specific training programme that included information about typical features of the different inflammatory skin diseases. The imaging acquisition had to be performed according to a standardized protocol. The inflammatory conditions were divided into three major groups: spongiotic, interface and psoriasiform dermatitis. Only histologically proven lesions were included. Exclusion criteria were areas with scales and scarring, some locations such as the scalp, as well as palmoplantar lesions.

One hundred and ninety-two cases were assessed using the standardized protocol to ensure a comparable high image quality. Nevertheless, 37 cases had to be excluded because of artefacts, leaving a total of 155 cases. These images were evaluated by the local centre retrospectively with awareness of the histological diagnosis. They were then re-evaluated by the blinded central coordinator of the study.

Spongiosis and vesicles were the most common RCM features in spongiotic dermatitis, whereas epidermal thickening was highly associated with psoriasiform dermatitis. Interface dermatitis exhibited blurring of the dermo-epidermal junction. These findings correspond well with the typical histological characteristics of these entities. Because RCM is a microscopic method, it is comparable with histology regarding the resolution, but has a limited penetration depth. Additionally, RCM offers an en-face view in contrast to the cross-sectional view of histological slices. The RCM descriptors showed a high concordance between the centres and a good agreement with the blinded coordinator centre. Based on these results, the authors developed a decision-tree diagram as an algorithm to diagnose inflammatory skin diseases using RCM. Moreover, they defined a scoring system of possible findings for fast clinical application of the method.

There are some limitations that should be mentioned. The RCM measurements and the evaluation of images requires experience and time. Furthermore, scanning larger areas or multiple lesions is not convenient. Some locations, such as the scalp, eyelids or ears, cannot be reached by the technique because of artefacts caused by hairs, movement or because the imaging tip of the device is too large. Scales and crusts cause severe artefacts, necessitating treatment of inflammatory skin changes to minimize scales before imaging with RCM. On the other hand, the shape and type of scales may give further hints for the diagnosis in conventional histology (i.e. neutrophils in the stratum corneum for psoriasis, serum crusts for acute eczema, follicular hyperkeratosis for lupus erythematosus). By removing the stratum corneum, these characteristic features of disease will be missed by RCM. Additionally, 
deeper findings in the middle dermis are not displayed by RCM because of the limited penetration depth of the signal. Therefore, the technique allows detection of superficial changes only. It is not suitable for diagnosing deep inflammatory diseases such as panniculitis. Finally, the device is expensive; it is not reasonable or needful to buy an RCM device solely for diagnosing inflammatory conditions. However, if this diagnostic method is already in place for skin tumours, then it may be used for diagnosing other skin diseases, too. The major advantage of RCM compared with histology is that the method is noninvasive, and permits repetitive measurements of the same lesion over time. This enables the monitoring of dynamic changes as well as assessing response to treatment. $^{11}$

In conclusion, Ardigo et al. ${ }^{1}$ present a well-designed multicentre study on the use of RCM in inflammatory skin diseases. This study shows that RCM may help to diagnose these diseases, to characterize them better, to evaluate treatment effects, and to monitor the healing process. The study is of great importance as it is likely to be the foundation stone for further research regarding in vivo investigation of inflammatory skin diseases and observing dynamic processes within the skin.

\section{Acknowledgments}

Priv.-Doz. Dr. med. Elke Sattler from the Ludwig Maximilian University of Munich, Germany, and Robert Pein kindly reviewed this manuscript.

\section{Conflicts of interest}

None declared.

Department of Dermatology, General Hospital

J. Welzel

Augsburg, Sauerbruchstrasse 6, 86179

Augsburg, Germany

E-mail: julia.welzel@klinikum-augsburg.de

\section{References}

1 Ardigo M, Longo C, Gonzalez S; the International Confocal Working Group Inflammatory Skin Diseases Project. Multicentre study on inflammatory skin diseases from The International Confocal Working Group: specific confocal microscopy features and an algorithmic method of diagnosis. Br J Dermatol 2016; 175:364 74 .

2 Pellacani G, Guitera P, Longo C et al. The impact of in vivo reflectance confocal microscopy for the diagnostic accuracy of melanoma and equivocal melanocytic lesions. J Invest Dermatol 2007; 127:2759-65.

3 Nori S, Rius-Díaz F, Cuevas J et al. Sensitivity and specificity of reflectance-mode confocal microscopy for in vivo diagnosis of basal cell carcinoma: a multicenter study. J Am Acad Dermatol 2004; 51:923-30.

4 Ulrich M, Maltusch A, Rius-Diaz F et al. Clinical applicability of in vivo reflectance confocal microscopy for the diagnosis of actinic keratoses. Dermatol Surg 2008; 34:610-9.

5 Maier T, Cekovic D, Ruzicka T et al. Treatment monitoring of topical ingenol mebutate in actinic keratoses with the combination of optical coherence tomography and reflectance confocal microscopy: a case series. Br J Dermatol 2015; 172:816-8.

6 Rothmund G, Sattler EC, Kaestle R et al. Confocal laser scanning microscopy as a new valuable tool in the diagnosis of onychomycosis - comparison of six diagnostic methods. Mycosis 2013; 56:47-55.

7 Sattler EC, Maier T, Hoffmann VS et al. Noninvasive in vivo detection and quantification of Demodex mites by confocal laser scanning microscopy. Br J Dermatol 2012; 167:1042-7.

8 Koller S, Gerger A, Ahlgrimm-Siess V et al. In vivo reflectance confocal microscopy of erythematosquamous skin diseases. Exp Dermatol 2009; 18:536-40.

9 Astner S, González S, Gonzalez E. Noninvasive evaluation of allergic and irritant contact dermatitis by in vivo reflectance confocal microscopy. Dermatitis 2006; 17:182-91.

10 Wolberink EA, van Erp PE, Teussink MM et al. Cellular features of psoriatic skin: imaging and quantification using in vivo reflectance confocal microscopy. Cytometry B Clin Cytom 2011; 80:141-9.

11 Hoogedoorn L, Peppelman M, van de Kerkhof PC et al. The value of in vivo reflectance confocal microscopy in the diagnosis and monitoring of inflammatory and infectious skin diseases: a systematic review. Br J Dermatol 2015; 172:1222-48. 